\title{
Association between change in HDL-C and vascular events in patients treated with statins: Report from the UK general practice research database
}

\author{
Lori D. Bash ${ }^{1}$, Tun-Ying Hsu ${ }^{2}$, Vasilisa Sazonov ${ }^{1}$, Baishali Ambegaonkar ${ }^{1}$ \\ ${ }^{1}$ Global Health Outcomes, Merck \& Co., Inc., Whitehouse Station, USA \\ ${ }^{2}$ Department of Epidemiology, University of Medicine and Dentistry of New Jersey, Piscataway, USA \\ Email: tun-ying.hsu@merck.com
}

Received 13 January 2012; revised 17 February 2012; accepted 25 February 2012

\begin{abstract}
Dyslipidemia, including low levels of high-density lipoprotein cholesterol (HDL-C), is a relatively wellestablished risk factor for cardiovascular disease. However, the independent association between changes in HDL-C and the subsequent risk of cardiovascular events has not been well studied. The retrospective cohort analysis was conducted to evaluate the association between changes in HDL-C and cardiovascular (CV) and/or cerebrovascular (CB) events among statin-treated patients. Patient demographics, clinical characteristics, laboratory data, and $\mathrm{CV} / \mathrm{CB}$ events, were collected from the UK General Practice Research Database. The association between the risk of an incident event and changes in patients' HDL-C was estimated using multivariate Cox proportional hazards models. Among 17,923 statin-treated patients with an average follow-up of 1.9 years, there were 815 $\mathrm{CV}$ events and $220 \mathrm{CB}$ events. The average change in HDL-C experienced was $0.4 \mathrm{mg} / \mathrm{dL}$, ranging from 11 $\mathrm{mg} / \mathrm{dL}$ average decrease in the lowest change quartile to $12 \mathrm{mg} / \mathrm{dL}$ average increase in the highest change quartile. CV events occurred at an average overall rate of 21 per 1000 person-years and 17 per 1000 person-years among individuals in the highest quartile of change in HDL-C levels. Fully adjusted Cox regression estimated a $6 \%$ decrease in hazards (HR, $0.94 ; 95 \%$ CI, $0.90,0.98$ ) of a subsequent $\mathrm{CV}$ event associated with each $5 \mathrm{mg} / \mathrm{dL}$ increase in HDL-C. Similar results (HR: $0.95,95 \%$ CI, $0.92,0.98$ ) were observed when assessing the association with changes in HDL-C and the composite outcome of $\mathrm{CV} / \mathrm{CB}$ event. Among statin-treated patients from UK clinical practices, increases in HDL-C were associated with a significantly decreased relative risk of experiencing $\mathrm{CV} / \mathrm{CB}$ events with a more pronounced effect on $\mathrm{CV}$ events. Therefore, a more aggressive treatment to in-
\end{abstract}

crease HDL-C could benefit patients by reducing the risk of $\mathrm{CV} / \mathrm{CB}$ events.

Keywords: LDL-C; HDL-C; CV and CB Event and LMT

\section{INTRODUCTION}

Cardiovascular disease is the world's leading cause of death, responsible for taking the lives 17.3 million individuals in 2008, counting $30 \%$ of all global deaths [1]. In 2008, over one-third of all deaths in the United Kingdom were attributable to cardiovascular diseases (CVD) [2], proportions very similar to those in the United States [3, 4], where in 2009, disease of heart, cerebrovascular disease, essential hypertension and hypertensive renal disease were ranked as $1^{\text {st }}, 4^{\text {th }}$ and $13^{\text {th }}$ cause of death respectively [5].

Several modifiable factors, among them lipids, contribute to the risk of cardiovascular disease [6]. Both US and European recommendations focus on low-density lipoprotein cholesterol (LDL-C) to lower cardiovascular risk $[7,8]$. However, both elevated triglycerides $[9,10]$, and low high-density lipoprotein cholesterol (HDL-C) $[11,12]$ have been identified as cardiovascular risk factors and have been shown to be associated with coronary events, even among patients treated with statins [13-15].

It is well established that dyslipidemia is directly related to atherosclerosis and one of the major risk factors of cardiovascular morbidity and mortality [16]. Among them, low levels of high-density lipoprotein cholesterol (HDL-C) have been shown to be associated with increased risk of several different adverse events $[11,12$, 17]. Specifically, HDL-C has been shown to be inversely associated with risk of CHD [11,12,17], as well as with CHD death and congestive heart failure [17] cardiac [18], cardiovascular [19], and all-cause mortality [18,19].

Increased HDL-C has been shown to be associated 
with reduced cardiovascular risk among several different populations including community-based middle aged whites of European descent [11,12,17,19], community based blacks [12], patients with optimal LDL-C levels $[18,20]$ and a history of coronary interventions $[18,20]$. Low HDL-C has been demonstrated to be predictive of major cardiac events [21], and one-year mortality [20] among CAD patients, as well as of cardiovascular (CV) events and total mortality in combination with elevated triglycerides [22].

Several observational studies have shown dyslipidemia, including low levels of HDL-C to be a relatively well-established risk factor for cardiovascular disease. Previous studies such as the lovastatin-treated arm of the AFCAPS/TexCAPS clinical trial and TNT trial also demonstrated a reduced risk of acute major coronary events associated with an increase in HDL-C [23,24].

However, while clinical trials [23] and observational studies [25], have shown increases in HDL-C to be associated with reduced risk of acute cardiovascular events, the independent association between changes in HDL-C and risk of cardiovascular events has not yet been characterized among observational population samples.

With $22 \%$ of its population over the age of 60 [3], a population at increased risk for cardiovascular disease, and one of the highest proportions in the world [3], further study of cardiovascular disease is of particular relevance to the United Kingdom. In this study, we evaluate the association between changes in HDL-C and CV and/ or cerebrovascular (CB) events among patients on statin treatment among a large, longitudinal, observation study in the UK.

\section{METHODS}

\subsection{Database}

The UK GPRD (General Practice Research Database) is a large computerized database that contains longitudinal representative clinical information on patient populations of general practices in the United Kingdom [26].

The GPRD contains data (including patient demographic information, details on GP visits, diagnoses from specialists' referrals and hospital admissions, prescriptions by GPs, and results of laboratory tests) on over 3.5 million patients. Systematically recorded since 1987 by UK general practitioners (GPs), data are sent anonymously to the Medicines and Healthcare Products Regulatory Agency, which collects and organizes this information for use by researchers. The GPRD database has been validated for use in research on health services and is extensively used in outcomes research [27].

Of this large database, over 200,000 patients were on at least one lipid-modifying therapy (LMT) between the years 1998-2005. Inclusion criteria captured all patients who were on lipid modifying therapy and further limited them to be adults with complete follow-up and minimum relevant data (for adjustment variables). Eligible patients among these included adults over age 35 receiving their first statin prescription between the beginning of 2000 and the end 2004, at least 6 weeks of statin monotherapy (no concomitant LMT) free of a $\mathrm{CV} / \mathrm{CB}$ event, as well as a valid and continuous database history for $>2$ years before the index date, including laboratory data from 1 year before to $\geq 1$ year after this date. These data included at least one complete lipid profile both before and after initiating statin therapy as well as a valid blood-pressure reading. A complete lipid profile panel consisted of measurements of total cholesterol (TC), LDL-C, HDL-C, and TGs recorded on the same day: either the index date or within 1 year before this date, as well as a follow-up profile from $\geq 42$ days after the index date (to allow sufficient time for LMT to take effect [28]). To assess the prospective association between change in HDL-C and incident events, subjects who may have experienced an event prior to the follow-up lipid measure were also excluded.

The entire follow-up period was up to 5 years. Only patient records derived from serology-linked practices (defined as practices from which available laboratory information was automatically coded and entered into the GPRD database) were eligible for analyses. Such practices were identified according to an algorithm based on the laboratory test data for potassium.

Baseline demographic information, including age and gender, were extracted from medical records. Clinical characteristics included body mass index (BMI), systolic blood pressure and the presence of hypertension (blood pressure $>140 / 90 \mathrm{mmHg}$ ); smoking status; history of comorbid conditions including diabetes mellitus diagnosis and treatment, ischemic heart disease, and prior $\mathrm{CB}$ events; and values for TC, LDL-C, HDL-C, and TG. Baseline serum TC, LDL-C, HDL-C, and TG levels were determined for each patient using the last available complete lipid profile before the index date. During the follow-up period, the serum lipids ((TC, LDL-C, HDL-C, and $\mathrm{TG}$ ) taken at a time closest to and prior to the $\mathrm{CV} /$ $\mathrm{CB}$ event) were considered as the follow-up measures for patients with an event. For patients without any event, the last lipid measurement during the follow-up period will be used.

Incident $\mathrm{CV}$ event was the primary outcome of interest. However, a composite outcome of either a $\mathrm{CV}$ or $\mathrm{CB}$ event, as well as an incident $\mathrm{CB}$ event was also assessed as a secondary endpoint of interest. CV events included $\mathrm{MI}$, angina pectoris, and coronary revascularization; while $\mathrm{CB}$ events included ischemic stroke and transient ischemic attacks. To ensure correct coding of events, both FREAD and OXMIS codes were used to enter diagnostic 
information related to $\mathrm{CV} / \mathrm{CB}$ events. The codes were run by certified practitioners and verified for accuracy (codes attached in the appendix).

\subsection{Statistical Analyses}

Analyses were limited to 17,923 patients meeting all inclusion criteria (Figure 1) aforementioned. All analyses were conducted using STATA $^{\circledR}$ software (StataCorp, College Station, TX).

Lipids were treated as continuous variables as well as categorized into quartiles for some analyses. Demographic and health characteristics of individuals categorized in different quartiles of change in HDL-C were compared. Kaplan-Meier survival estimates were plotted by quartiles of HDL-C and differences in survival across quartiles were tested by log-rank tests. Proportional hazards regression was used to estimate unadjusted and adjusted hazard ratios (HRs) and 95\% confidence intervals

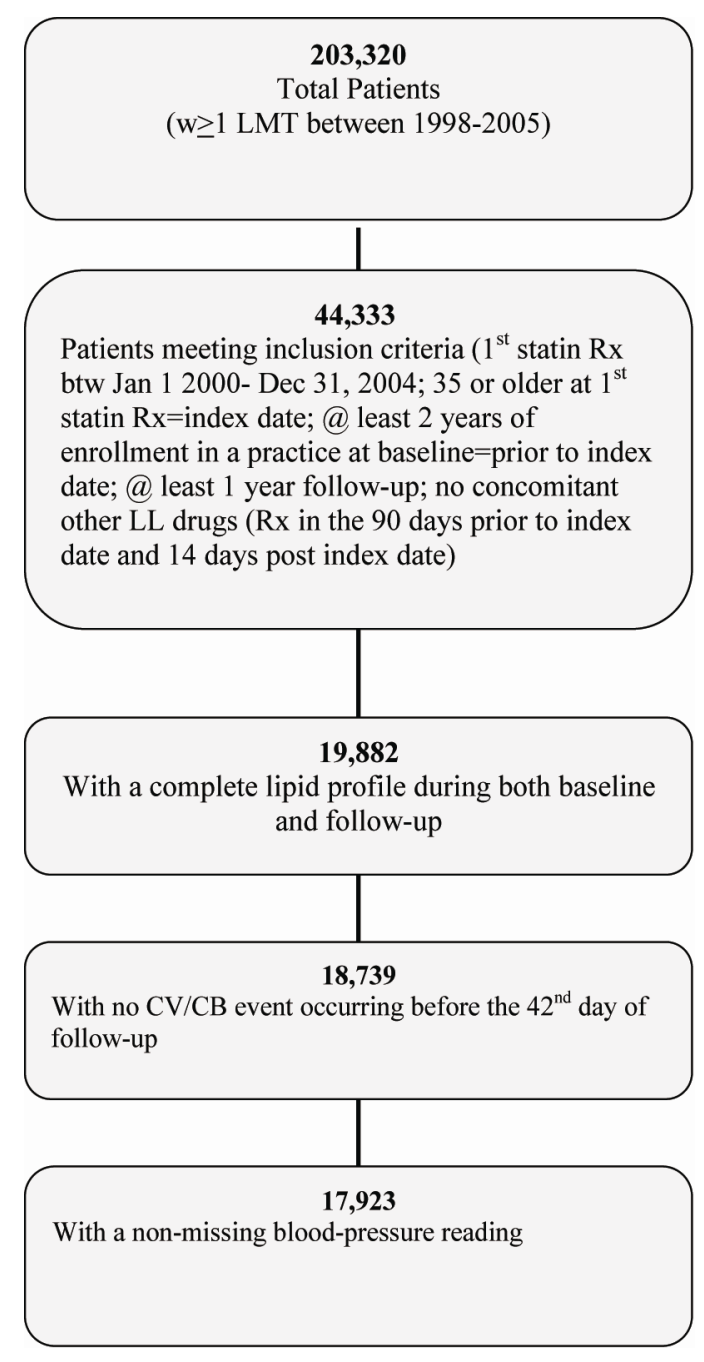

Figure 1. Flowchart of patient selection/inclusion/exclusion criteria.
(CIs) associated with a 5 unit increase in HDL-C. Fully adjusted multivariate adjusted models included changes in lipids, baseline lipid variables, age, sex, medical history (binary variables accounting for prevalent cardiovascular disease, diabetes, hypertension), baseline blood pressure, use of antihypertensives, body mass index, smoking status, year of statin initiation, mean statin dose and medication possession ratio.

\section{RESULTS}

After an average follow-up time of 1.9 years, a total of $1,035 \mathrm{CV} / \mathrm{CB}$ ( $815 \mathrm{CV}$ and $220 \mathrm{CB}$ events) events were experienced among 17,923 patients who took statins over a total of 33,392 person-years.

Characteristics of the study population overall, and by eventual progression to a CV event are delineated in Table 1. Characteristics of participants by change in HDL$\mathrm{C}$ are delineated in Table 2. At baseline, persons who subsequently developed a cardiovascular event were more likely to be older, have lower lipid levels (HDL-C, LDL$\mathrm{C}$ and triglycerides), lower BMI, lower systolic blood pressure, be on antihypertensive medications, be male, have prevalent cardiovascular disease, be former smokers, and have prevalent diabetes or hypertension at baseline (Table 1).

Baseline characteristics of study participants in the highest quartile of change in HDL-C levels (quartile 4) were similar to those of the rest of the sample though they did have lower baseline HDL-C levels and higher LDL-C and triglyceride levels, spent a longer duration of time on statins, were more likely to be female and less likely to have prevalent diabetes at baseline (Table 2).

Individuals experiencing the greatest increases in HDL$\mathrm{C}$ (quartile 4) also experienced the lowest incidence rates of $\mathrm{CV}$ events with a rate of 16.9 per 1000 person-years, (Table 3) and those experiencing the least amount of increase in HDL-C (quartile 1), the greatest rate of CV events at a rate of 22.1 per 1000 person-years. While there was a statistically significant linear trend $(p=0.008)$ demonstrating a progressive increase between all four quartiles, the larger difference in rate was observed between those experiencing changes in HDL-C above the $75^{\text {th }}$ percentile (quartile 4), from the rest of the sample (p $<0.001$, Table 3). Similar trends were observed for the composite outcome of $\mathrm{CV}$ or $\mathrm{CB}$ events, but not for $\mathrm{CB}$ event alone. In looking at the four quartiles individually and when comparing the $4^{\text {th }}$ quartile to the rest of the sample, both linear trends across quartiles and significant differences between the highest quartile and the remaining were statistically significant for the composite (CV/ $\mathrm{CB})$ outcoome ( $\mathrm{p}=0.002$ and $\mathrm{p}<0.001$, respectively), but not for CB events alone $(\mathrm{p}=0.301$ and $\mathrm{p}=0.451$, respectively). 
Table 1. Participant sample characteristics, overall and subsequent outcome status.

\begin{tabular}{|c|c|c|c|c|c|c|c|}
\hline & \multicolumn{2}{|c|}{ Overall } & \multicolumn{2}{|c|}{ No event } & \multicolumn{2}{|c|}{ CV event } & \multirow{2}{*}{ p-value } \\
\hline & Mean & SD & Mean & SD & Mean & SD & \\
\hline $\mathbf{N}$ & \multicolumn{2}{|c|}{17,923} & \multicolumn{2}{|c|}{17,108} & \multicolumn{2}{|c|}{815} & \\
\hline Age in Years & 65.7 & 10.4 & 65.6 & 10.5 & 68.5 & 9.3 & $<0.0001$ \\
\hline Baseline HDL-C (mg/dL) & 55.1 & 16.3 & 55.2 & 16.3 & 52.8 & 14.4 & $<0.0001$ \\
\hline Follow-up HDL-C (mg/dL) & 55.5 & 16.0 & 55.6 & 16.1 & 52.7 & 14.9 & $<0.0001$ \\
\hline Change in HDL-C (mg/dL) & 0.4 & 9.6 & 0.4 & 9.7 & -0.1 & 8.2 & 0.1675 \\
\hline Change in HDL-C (\%) & $0.7 \%$ & & $0.8 \%$ & & $-0.1 \%$ & & \\
\hline Baseline LDL-C (mg/dL) & 162.3 & 39.3 & 162.6 & 39.3 & 156.4 & 37.7 & $<0.0001$ \\
\hline Follow-up LDL-C (mg/dL) & 96.5 & 33.8 & 96.7 & 34.0 & 91.8 & 29.3 & $<0.0001$ \\
\hline Change in LDL-C (mg/dL) & -65.8 & 38.1 & -65.9 & 38.2 & -64.6 & 35.6 & 0.3514 \\
\hline Change in LDL-C (\%) & $-40.6 \%$ & & $-40.5 \%$ & & $-41.3 \%$ & & \\
\hline Baseline Triglycerides (mg/dL) & 172.5 & 80.0 & 173.0 & 80.4 & 163.0 & 69.9 & 0.0005 \\
\hline Follow-up Triglycerides (mg/dL) & 137.2 & 64.7 & 137.4 & 64.8 & 131.8 & 61.3 & 0.0147 \\
\hline Change in Triglycerides $(\mathrm{mg} / \mathrm{dL})$ & -35.3 & 61.4 & -35.5 & 61.7 & -31.2 & 55.4 & 0.0506 \\
\hline Change in Triglycerides (\%) & $-20.5 \%$ & & $-20.5 \%$ & & $-19.1 \%$ & & \\
\hline Baseline Systolic Blood Pressure & 147.1 & 17.2 & 147.2 & 17.2 & 145.2 & 18.0 & 0.0015 \\
\hline Body Mass Index $\left(\mathrm{kg} / \mathrm{m}^{2}\right)$ & 28.1 & 5.0 & 28.1 & 5.1 & 27.6 & 4.4 & 0.0020 \\
\hline Time on Statin (FOLLOW-UP YEARS) & 1.9 & 1.0 & 2.2 & 1.0 & 1.0 & 0.8 & $<0.0001$ \\
\hline Medication Possession Ratio ${ }^{\dagger}$ & 0.9 & 0.2 & 0.9 & 0.2 & 1.1 & 0.3 & $<0.0001$ \\
\hline Antihypertensive Medications (ANYTIME) & 34.4 & 44.3 & 34.0 & 44.2 & 42.8 & 47.2 & $<0.0001$ \\
\hline \multirow[t]{2}{*}{ Antihypertensive Medications (1 YR PRIOR) } & 5.7 & 5.6 & 5.7 & 5.6 & 6.6 & 5.3 & $<0.0001$ \\
\hline & $\mathbf{N}$ & $\%$ & $\mathbf{N}$ & $\%$ & $\mathbf{N}$ & $\%$ & p-value \\
\hline Female & 8822 & $49.2 \%$ & 8502 & $49.7 \%$ & 320 & $39.3 \%$ & $<0.0001$ \\
\hline Prevalent Cardiovascular Disease & 4267 & $23.8 \%$ & 3676 & $21.5 \%$ & 591 & $72.5 \%$ & $<0.0001$ \\
\hline Prevalent Cerebrovascular Disease & 2062 & $11.5 \%$ & 1965 & $11.5 \%$ & 97 & $11.9 \%$ & 0.7161 \\
\hline Prevalent Diabetes & 5502 & $30.7 \%$ & 5356 & $31.3 \%$ & 146 & $17.9 \%$ & $<0.0001$ \\
\hline Prevalent Hypertension & 10,006 & $55.8 \%$ & 9599 & $56.1 \%$ & 407 & $49.9 \%$ & 0.0005 \\
\hline \multicolumn{8}{|l|}{ Smoking } \\
\hline Current Smoker & 2,901 & $16.2 \%$ & 2783 & $16.3 \%$ & 118 & $14.5 \%$ & 0.1756 \\
\hline Never Smoked & 9,117 & $50.9 \%$ & 8753 & $51.2 \%$ & 364 & $44.7 \%$ & 0.0003 \\
\hline Ex-smoker & 5,370 & $30.0 \%$ & 5072 & $29.6 \%$ & 298 & $36.6 \%$ & $<0.0001$ \\
\hline Missing & 535 & $3.0 \%$ & 500 & $2.9 \%$ & 35 & $4.3 \%$ & 0.0245 \\
\hline \multicolumn{8}{|l|}{ Index Year of Statin Initiation } \\
\hline 2000 & 765 & $4.3 \%$ & 666 & $3.9 \%$ & 99 & $12.1 \%$ & $<0.0001$ \\
\hline 2001 & 2118 & $11.8 \%$ & 1934 & $11.3 \%$ & 184 & $22.6 \%$ & $<0.0001$ \\
\hline 2002 & 3554 & $19.8 \%$ & 3332 & $19.5 \%$ & 222 & $27.2 \%$ & $<0.0001$ \\
\hline 2003 & 6769 & $37.8 \%$ & 6556 & $38.3 \%$ & 213 & $26.1 \%$ & $<0.0001$ \\
\hline 2004 & 4717 & $26.3 \%$ & 4620 & $27.0 \%$ & 97 & $11.9 \%$ & $<0.0001$ \\
\hline Antihypertensive Medications (ANYTIME) & 617,055 & & 582,192 & & 34,863 & & \\
\hline Antihypertensive Medications (1 YR PRIOR) & 102,257 & & 96,916 & & 5341 & & \\
\hline
\end{tabular}

${ }^{\dagger}$ Medication Possession Ratio calculated as: the sum of the days supplied divided by follow-up time.

Kaplan-Meier plots by quartile of change in HDL-C are shown in Figure 2. Log-rank tests results demon- strate evidence that progressively higher quartiles of change in HDL-C were associated with reduced risk of 
Table 2. Participant sample characteristics, overall and by change in HDL-C.

\begin{tabular}{|c|c|c|c|c|c|c|c|}
\hline & & & \multicolumn{5}{|c|}{ Quartiles of Change in HDL-C (mg/dL) Quartiles } \\
\hline & \multirow{2}{*}{\multicolumn{2}{|c|}{ Overall }} & \multirow{2}{*}{\multicolumn{2}{|c|}{$\begin{array}{c}Q 1-Q 3 \\
-144 \text { to }+5.4\end{array}$}} & \multicolumn{2}{|c|}{ Q4 } & \multirow[b]{3}{*}{$\mathrm{p}$-Value } \\
\hline & & & & & \multicolumn{2}{|c|}{$\geq+5.4$ to +120.5} & \\
\hline & Mean & SD & Mean & SD & Mean & SD & \\
\hline $\mathbf{N}$ & \multicolumn{2}{|c|}{17,923} & \multicolumn{2}{|c|}{13,386} & \multicolumn{2}{|c|}{4,537} & \\
\hline Age in Years & 65.7 & 10.4 & 65.7 & 10.5 & 65.8 & 10.3 & 0.7274 \\
\hline Baseline HDL-C (mg/dL) & 55.1 & 16.3 & 56.0 & 16.5 & 52.3 & 15.2 & $<0.0001$ \\
\hline Follow-up HDL-C (mg/dL) & 55.5 & 16.0 & 52.6 & 14.6 & 64.0 & 17.0 & $<0.0001$ \\
\hline Change in HDL-C (mg/dL) & 0.4 & 9.6 & -3.4 & 7.3 & 11.7 & 6.3 & $<0.0001$ \\
\hline Change in HDL-C (\%) & $0.7 \%$ & & $-6.1 \%$ & & $22.3 \%$ & & \\
\hline Baseline LDL-C-C (mg/dL) & 162.3 & 39.3 & 161.6 & 38.9 & 164.4 & 40.2 & $<0.0001$ \\
\hline Follow-up LDL-C (mg/dL) & 96.5 & 33.8 & 96.3 & 33.7 & 97.0 & 34.3 & 0.2642 \\
\hline Change in LDL-C (mg/dL) & -65.8 & 38.1 & -65.3 & 37.6 & -67.4 & 39.6 & 0.0013 \\
\hline Change in LDL-C (\%) & $-40.6 \%$ & & $-40.4 \%$ & & $-41.0 \%$ & & \\
\hline Baseline Triglycerides (mg/dL) & 172.5 & 80.0 & 170.7 & 79.8 & 177.8 & 80.5 & $<0.0001$ \\
\hline Follow-up Triglycerides (mg/dL) & 137.2 & 64.7 & 140.0 & 65.8 & 129.0 & 60.4 & $<0.0001$ \\
\hline Change in Triglycerides $(\mathrm{mg} / \mathrm{dL})$ & -35.3 & 61.4 & -30.8 & 60.4 & -48.7 & 62.5 & $<0.0001$ \\
\hline Change in Triglycerides (\%) & $-20.5 \%$ & & $-18.0 \%$ & & $-27.4 \%$ & & \\
\hline Baseline Systolic Blood Pressure & 147.1 & 17.2 & 147.0 & 17.3 & 147.4 & 17.0 & 0.1821 \\
\hline Body Mass Index $\left(\mathrm{kg} / \mathrm{m}^{2}\right)$ & 28.1 & 5.0 & 28.1 & 5.1 & 28.0 & 4.9 & 0.1459 \\
\hline Time on Statin (FOLLOW-UP YEARS) & 1.9 & 1.0 & 1.8 & 1.0 & 2.0 & 1.1 & $<0.0001$ \\
\hline Medication Possession Ratio ${ }^{\dagger}$ & 0.9 & 0.2 & 0.9 & 0.2 & 1.0 & 0.2 & 0.0001 \\
\hline Antihypertensive Medications (Anytime) & 34.4 & 44.3 & 34.1 & 44.3 & 35.3 & 44.5 & 0.1389 \\
\hline \multirow[t]{2}{*}{ Antihypertensive Medications (1 year prior) } & 5.7 & 5.6 & 5.7 & 5.6 & 5.9 & 5.5 & 0.0254 \\
\hline & $\mathbf{N}$ & $\%$ & $\mathbf{N}$ & $\%$ & $\mathbf{N}$ & $\%$ & $\mathrm{p}$-Value \\
\hline Female & 8822 & $49.2 \%$ & 6415 & $47.9 \%$ & 2407 & $53.1 \%$ & $<0.0001$ \\
\hline Prevalent Cardiovascular Disease & 4267 & $23.8 \%$ & 3213 & $24.0 \%$ & 1054 & $23.2 \%$ & 0.2917 \\
\hline Prevalent Cerebrovascular Disease & 2062 & $11.5 \%$ & 1509 & $11.3 \%$ & 553 & $12.2 \%$ & 0.0948 \\
\hline Prevalent Diabetes & 5502 & $30.7 \%$ & 4171 & $31.2 \%$ & 1331 & $29.3 \%$ & 0.0214 \\
\hline Prevalent Hypertension & 10,006 & $55.8 \%$ & 7437 & $55.6 \%$ & 2569 & $56.6 \%$ & 0.2118 \\
\hline \multicolumn{8}{|l|}{ Smoking } \\
\hline Current Smoker & 2901 & $16.2 \%$ & 2126 & $15.9 \%$ & 775 & $17.1 \%$ & 0.0580 \\
\hline Never Smoked & 9117 & $50.9 \%$ & 6854 & $51.2 \%$ & 2263 & $49.9 \%$ & 0.1232 \\
\hline Ex-smoker & 5370 & $30.0 \%$ & 4009 & $29.9 \%$ & 1361 & $30.0 \%$ & 0.9508 \\
\hline Missing & 535 & $3.0 \%$ & 397 & $3.0 \%$ & 138 & $3.0 \%$ & 0.7952 \\
\hline \multicolumn{8}{|l|}{ Index Year of Statin Initiation } \\
\hline 2000 & 765 & $4.3 \%$ & 541 & $4.0 \%$ & 224 & $4.9 \%$ & 0.0099 \\
\hline 2001 & 2118 & $11.8 \%$ & 1430 & $10.7 \%$ & 688 & $15.2 \%$ & $<0.0001$ \\
\hline 2002 & 3554 & $19.8 \%$ & 2593 & $19.4 \%$ & 961 & $21.2 \%$ & 0.0082 \\
\hline 2003 & 6769 & $37.8 \%$ & 5142 & $38.4 \%$ & 1627 & $35.9 \%$ & 0.0022 \\
\hline 2004 & 4717 & $26.3 \%$ & 3680 & $27.5 \%$ & 1037 & $22.9 \%$ & $<0.0001$ \\
\hline Antihypertensive Medications (Anytime) & 617,055 & & 457,034 & & 160,021 & & \\
\hline Antihypertensive Medications (1 year prior) & 102,257 & & 75,647 & & 26,610 & & \\
\hline
\end{tabular}

${ }^{\dagger}$ Medication Possession Ratio calculated as: the sum of the days supplied divided by follow-up time. 
Table 3. Incident rates of cardiovascular and cerebrovascular events, by change in HDL-C quartiles.

\begin{tabular}{|c|c|c|c|c|c|}
\hline & \multicolumn{5}{|c|}{ Quartiles of Change in HDL-C (mg/dL) Quartiles } \\
\hline & Overall & $\begin{array}{c}\text { Q1 } \\
-144 \text { to }-4\end{array}$ & $\begin{array}{c}Q 2 \\
\geq-4 \text { to } 0\end{array}$ & $\begin{array}{c}\mathrm{Q3} \\
\geq 0 \text { to }+5.4\end{array}$ & $\begin{array}{c}Q 4 \\
\geq+5.4 \text { to }+120.5\end{array}$ \\
\hline \multicolumn{6}{|c|}{ CV EVENT ONLY } \\
\hline$\#$ of events/N & $\begin{array}{c}815 / 17,923 \\
(4.55 \%)\end{array}$ & $\begin{array}{c}200 / 4338 \\
(4.61 \%)\end{array}$ & $\begin{array}{c}166 / 3232 \\
(5.14 \%)\end{array}$ & $\begin{array}{c}273 / 5816 \\
(4.69 \%)\end{array}$ & $\begin{array}{c}176 / 4537 \\
(3.88 \%)\end{array}$ \\
\hline Incidence Rates per 1000 p-years $(95 \% \mathrm{CI})$ & $\begin{array}{c}21.11 \\
(19.67,22.57)\end{array}$ & $\begin{array}{c}22.06 \\
(19.00,25.12)\end{array}$ & $\begin{array}{c}24.53 \\
(20.80,28.26)\end{array}$ & $\begin{array}{c}22.12 \\
(19.50,24.74)\end{array}$ & $\begin{array}{c}16.89 \\
(14.39,19.38)\end{array}$ \\
\hline p-trend & \multicolumn{5}{|c|}{0.008} \\
\hline & \multicolumn{3}{|c|}{$\begin{array}{c}\text { Q1 - Q3 } \\
-144 \text { to }+5.4\end{array}$} & \multicolumn{2}{|c|}{$\begin{array}{c}\mathrm{Q4} \\
\geq+5.4 \text { to }+120.5\end{array}$} \\
\hline$\#$ of events/N & & \multicolumn{2}{|c|}{$\begin{array}{c}639 / 13,386 \\
(4.77 \%)\end{array}$} & \multicolumn{2}{|c|}{$\begin{array}{c}176 / 4537 \\
(3.88 \%)\end{array}$} \\
\hline Incidence Rates per 1000 p-years $(95 \% \mathrm{CI})$ & & \multicolumn{2}{|c|}{$\begin{array}{c}22.68 \\
(20.92,24.44)\end{array}$} & \multicolumn{2}{|c|}{$\begin{array}{c}16.89 \\
(14.39,19.38)\end{array}$} \\
\hline p-difference & \multicolumn{5}{|c|}{0.001} \\
\hline \multicolumn{6}{|c|}{ OVERALL (CV OR CB EVENT) } \\
\hline$\#$ of events/N & $\begin{array}{c}1035 / 17,923 \\
(5.77 \%)\end{array}$ & $\begin{array}{c}805 / 4338 \\
(5.86 \%)\end{array}$ & $\begin{array}{c}212 / 3232 \\
(6.56 \%)\end{array}$ & $\begin{array}{c}339 / 5816 \\
(5.83 \%)\end{array}$ & $\begin{array}{c}230 / 4537 \\
(5.07 \%)\end{array}$ \\
\hline Incidence Rates per 1000 p-years $(95 \% \mathrm{CI})$ & $\begin{array}{c}31.00 \\
(29.16,32.94)\end{array}$ & $\begin{array}{c}32.35 \\
(28.61,36.58)\end{array}$ & $\begin{array}{c}36.82 \\
(32.19,42.13)\end{array}$ & $\begin{array}{c}32.05 \\
(28.81,35.65)\end{array}$ & $\begin{array}{c}24.99 \\
(21.96,28.43)\end{array}$ \\
\hline p-trend & \multicolumn{5}{|c|}{0.002} \\
\hline & & \multicolumn{2}{|c|}{$\begin{array}{c}Q 1-Q 3 \\
-144 \text { to }+5.4\end{array}$} & \multicolumn{2}{|c|}{$\begin{array}{c}\mathrm{Q4} \\
\geq+5.4 \text { to }+120.5\end{array}$} \\
\hline$\#$ of events/N & & \multicolumn{2}{|c|}{$\begin{array}{c}805 / 13,386 \\
(6.01 \%)\end{array}$} & \multicolumn{2}{|c|}{$\begin{array}{c}230 / 4537 \\
(5.07 \%)\end{array}$} \\
\hline Incidence Rates per 1000 p-years $(95 \% \mathrm{CI})$ & & \multicolumn{2}{|c|}{$\begin{array}{c}33.28 \\
(30.98,35.58)\end{array}$} & \multicolumn{2}{|c|}{$\begin{array}{c}24.99 \\
(21.96,28.43)\end{array}$} \\
\hline p-difference & \multicolumn{5}{|c|}{$<0.001$} \\
\hline \multicolumn{6}{|c|}{ CB EVENT ONLY } \\
\hline$\#$ of events/N & $\begin{array}{c}220 / 17,923 \\
(1.23 \%)\end{array}$ & $\begin{array}{l}54 / 4,338 \\
(1.24 \%)\end{array}$ & $\begin{array}{l}46 / 3232 \\
(1.42 \%)\end{array}$ & $\begin{array}{l}66 / 5816 \\
(1.13 \%)\end{array}$ & $\begin{array}{l}54 / 4537 \\
(1.19 \%)\end{array}$ \\
\hline Incidence Rates per 1000 p-years $(95 \% \mathrm{CI})$ & & $\begin{array}{c}5.79 \\
(4.24,7.33)\end{array}$ & $\begin{array}{c}6.58 \\
(4.68,8.48)\end{array}$ & $\begin{array}{c}5.16 \\
(3.91,6.40)\end{array}$ & $\begin{array}{c}5.07 \\
(3.71,6.42)\end{array}$ \\
\hline p-trend & \multicolumn{5}{|c|}{0.301} \\
\hline & & \multicolumn{2}{|c|}{$\begin{array}{c}\text { Q1 - Q3 } \\
-144 \text { to }+5.4\end{array}$} & \multicolumn{2}{|c|}{$\begin{array}{c}\mathrm{Q4} \\
\geq+5.4 \text { to }+120.5\end{array}$} \\
\hline$\#$ of events/N & & \multicolumn{2}{|c|}{$\begin{array}{c}166 / 13,386 \\
(1.24 \%)\end{array}$} & \multicolumn{2}{|r|}{$\begin{array}{l}54 / 4537 \\
(1.19 \%)\end{array}$} \\
\hline Incidence Rates per 1000 p-years $(95 \% \mathrm{CI})$ & & \multicolumn{2}{|c|}{$\begin{array}{c}5.70 \\
(4.83,6.57)\end{array}$} & \multicolumn{2}{|c|}{$\begin{array}{c}5.07 \\
(3.71,6.42)\end{array}$} \\
\hline p-difference & & & 0.451 & & \\
\hline
\end{tabular}

the composite $\mathrm{CV} / \mathrm{CB}$ events.

In both unadjusted, moderately and fully adjusted analyses (Table 4), changes in HDL-C were inversely asso- ciated with risk of both a subsequent $\mathrm{CV}$ event $(\mathrm{p}<0.01)$ and $\mathrm{CV} / \mathrm{CB}$ event $(\mathrm{p}<0.02)$. In all models, there was consistently approximately a $5 \%$ decrease hazards of a $\mathrm{CV}$ 


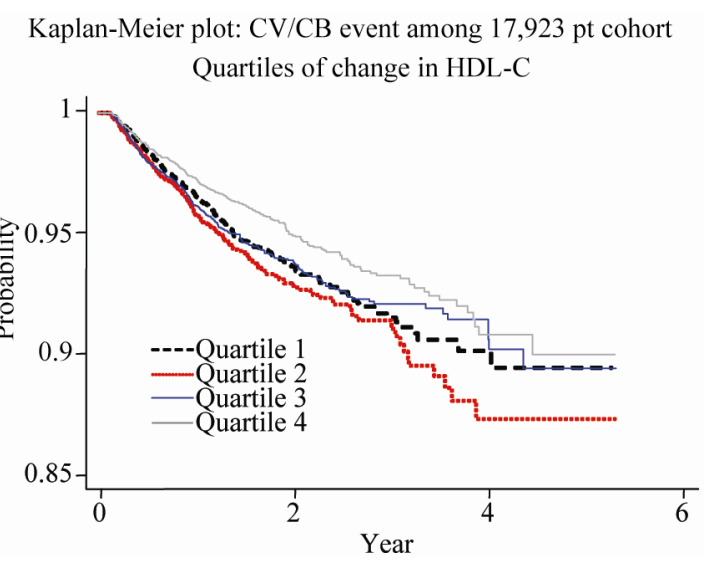

Kaplan-Meier plot: CV/CB event among 17,923 pt cohort Quartiles of change in HDL-C

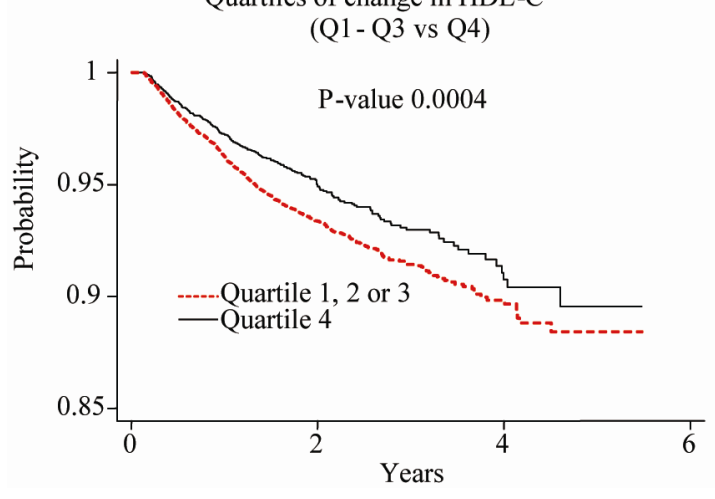

Figure 2. Kaplan-Meier Survival Estimate, Overall and by quartiles of change in HDL-C.

event associated with each $5 \mathrm{mg} / \mathrm{dL}$ increase in HDL-C. The $6 \%$ reduced risk of a $\mathrm{CV}$ event (HR, $0.94 ; 95 \% \mathrm{CI}$, $0.90,0.98 \%$ ) was present even after fully adjusting for changes in other lipids, baseline levels of all lipids, as well as statin treatment and comorbidities. The composite event was likewise consistently inversely related to changes in HDL-C, though there was no statistically significant association between changes in HDL-C and CB events individually.

\section{DISCUSSION}

The current study expands on previous findings which have demonstrated an inverse association between HDL$\mathrm{C}$ and risk of cardiovascular morbidities and mortality $[10,11,13,14,16-18]$. While confirming clinical trial based findings $[19,23]$, this retrospective observational study of UK primary care practices demonstrated increases in HDL-C to be associated with decreased risk of subsequent acute cardiovascular events among patients on statins.

Compared to other observational studies such as Grover et al. (2009) [29], which investigated the effects of increased levels of HDL-C among the Framingham Offspring cohort (which mainly consists of young adults), this study population consists of higher risk patients, with more than half having prevalent hypertension, and nearly a third, diabetes. Grover et al. [29] found that a 5 $\mathrm{mg} / \mathrm{dL}$ increase in HDL-C was associated with a $21 \%$ reduced hazard of a $\mathrm{CV}$ event. In the current study (of higher risk individuals) we observed individuals who attained a $5 \mathrm{mg} / \mathrm{dL}$ increase in HDL-C at follow-up to be associated with a $6 \%$ decreased hazard of experiencing a $\mathrm{CV}$ event. While we here demonstrated a lower reduction in risk gained among this high risk population (compared to the low risk populations previously reported), it is notable that observations demonstrate that even in this relatively high-risk population, patients can still reduce their risk of cardiovascular events by achieving increases in HDL-C.

Table 4: The association between changes in high-density lipoprotein cholesterol on cardiovascular and cerebrovascular events: results of cox proportional hazards regression.

\begin{tabular}{|c|c|c|c|c|c|c|c|c|c|c|c|c|}
\hline & \multicolumn{3}{|c|}{ 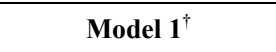 } & \multicolumn{3}{|c|}{ Model $2^{\ddagger}$} & \multicolumn{3}{|c|}{ Model 3a ${ }^{¥}$} & \multicolumn{3}{|c|}{ Model 4a } \\
\hline & HR & \multicolumn{2}{|r|}{$95 \%$ CI } & HR & \multicolumn{2}{|r|}{$95 \% \mathrm{CI}$} & \multirow[t]{2}{*}{ HR } & \multicolumn{2}{|r|}{$95 \%$ CI } & \multirow[t]{2}{*}{ HR } & \multicolumn{2}{|c|}{$95 \% \mathrm{CI}$} \\
\hline \multicolumn{11}{|c|}{ CV Event Only } & & \\
\hline Increase in HDL-C (per $5 \mathrm{mg} / \mathrm{dL}$ ) & $0.93^{\prime}$ & 0.90 & 0.97 & 0.95 & 0.91 & 0.98 & $0.95^{\prime \prime}$ & 0.91 & 0.99 & 0.94 & 0.90 & 0.98 \\
\hline Increase in LDL-C (per $10 \mathrm{mg} / \mathrm{dL}$ ) & $0.97^{\uparrow}$ & 0.94 & 0.99 & $0.97^{\#}$ & 0.95 & 1.00 & $1.05^{\natural}$ & 1.02 & 1.07 & 0.99 & 0.96 & 1.01 \\
\hline \multicolumn{13}{|c|}{ CV or CB Event } \\
\hline Increase in HDL-C (per $5 \mathrm{mg} / \mathrm{dL}$ ) & $0.95^{\natural}$ & 0.91 & 0.98 & $0.95^{\natural}$ & 0.92 & 0.99 & 0.96 & 0.93 & 0.99 & 0.95 & 0.92 & 0.98 \\
\hline Increase in LDL-C (per $10 \mathrm{mg} / \mathrm{dL}$ ) & 1.01 & 0.98 & 1.03 & 1.02 & 1.00 & 1.04 & $1.07^{\natural}$ & 1.04 & 1.09 & $1.03^{H}$ & 1.00 & 1.05 \\
\hline \multicolumn{13}{|c|}{ CB Event Only } \\
\hline Increase in HDL-C (per $5 \mathrm{mg} / \mathrm{dL}$ ) & 1.00 & 0.93 & 1.08 & 1.00 & 0.93 & 1.07 & 1.00 & 0.93 & 1.08 & 1.00 & 0.93 & 1.07 \\
\hline Increase in LDL-C (per $10 \mathrm{mg} / \mathrm{dL}$ ) & 0.94 & 0.90 & 0.99 & 0.96 & 0.92 & 1.01 & 1.02 & 0.97 & 1.08 & 0.98 & 0.93 & 1.03 \\
\hline
\end{tabular}

${ }^{\dagger}$ Model 1 adjusted for variables here as well as baseline HDL-C, LDL-C, TG. ${ }^{\ddagger}$ Model 2 adjusted for all in Model 1 as well as age, gender, prevalent CVD, prevalent diabetes, prevalent hypertension. ${ }^{\S}$ Model 4 adjusted for all in Model 2 as well as index year of statin initiation, mean statin dose per day, baseline SBP, BMI, smoking status (current, former, never, missing), and number of antihypertensives taken in prior year. ${ }^{¥}$ Model 3 adjusted for all in Model 4 as well as medication possession ratio ${ }^{*}$. Medication Possession Ratio calculated as: the sum of the days supplied divided by follow-up time. "p $<0.01$, ${ }^{p} \mathrm{p}<0.05$. 
This study assessed multiple lipid parameters and included extensive data for potential confounders. Among the other strengths of the present study are the large sample size, representative nature of the database, and systematic collection of data within it. While our study sample is not representative of the UK population, it is representative of patients in general practices and has been well validated for use in research on health services and is extensively used in outcomes research [21]. In addition, because exclusion criteria were applied to this sample, there is potential that our observations may no longer be representative of all patients in UK general practice. It is however, reasonable to assume that our observations can be generalized to patients in general practices who are on LMT.

As any other observational study, observations here may be prone to residual confounding of unmeasured factors. Specifically of note is that findings were apparent in a population of individuals where everyone was on statins, so it may not be possible to determine whether the benefit gained by these patients were attributable to changes in HDL-C alone, or if some additional benefit was conferred from the statin therapy itself.

Nevertheless, results were consistent with previous findings documenting an inverse association between HDL-C and risk of acute CV events [19]. Findings were less consistent with the association between changes in HDL-C and risk of subsequent CB events. While changes in HDL-C were inversely related to risk of the composite outcome of a CV/CB event, the observed association may have been driven by the cardiovascular events which made up nearly $80 \%$ of the total number of events. The lack of an association found between HDL-C and CB events alone, while potentially due to the limited number of events and the lack of power to detect an association, is consistent with existing literature, which has been inconsistent in demonstrating a relationship between stroke and dyslipidemia [30]. In fact, the lack of an observed association with $\mathrm{CB}$ events, because it contrasts with the observed association between changes in HDL-C and CV events, strengthens the likelihood that this association may not simply be an artifact of the data.

Observations from fully adjusted Cox proportional hazards models demonstrate a $5 \%$ decrease in risk of a subsequent $\mathrm{CV} / \mathrm{CB}$ event ( $6 \%$ for $\mathrm{CV}$ events only) for each $5 \mathrm{mg} / \mathrm{dL}$ increase in HDL-C. Among this population of patients receiving lipid-modifying therapy, this degree of increase in HDL-C is only experienced among approximately $25 \%$ of patients, while $50 \%$ of patients did not experience an increase in HDL-C at all (individuals in the bottom 2 quartiles of change in HDL-C experienced decreases in HDL-C or no change at all). These observations suggest that most patients who are already on lipid modifying therapy would benefit from further lipid-modifying treatment, specifically treatments which increase HDL-C levels.

We assessed the association between changes in HDL$\mathrm{C}$ and $\mathrm{CV}$ and/or $\mathrm{CB}$ events among patients on statin treatment among a large, longitudinal, observation study in the UK. We observed, even in this relatively high-risk population, in which all patients were on statin therapy at the start of the study, individuals who were able to achieve a sufficient increase in HDL-C at follow-up were conferred a decrease in risk of cardiovascular events. A significant linear relationship between increase in HDL-C and reduction in $\mathrm{CV} / \mathrm{CB}$ events can be quantified among high-risk population. Deeper investigation shows that the increase in HDL-C is beneficial for patients in decreasing $\mathrm{CV}$ events mainly and not so much for $\mathrm{CB}$ events (due to fewer $\mathrm{CB}$ events). The risk reduction of $5 \%$ in $\mathrm{CV} / \mathrm{CB}$ events associated with $5 \mathrm{mg} / \mathrm{dL}$ increase in HDL-C observed in this research is modest compared to previous studies due to the risk profile of study sample (high risk in current research vs. general population in earlier studies). In addition to existing literature which has demonstrated low levels of HDL-C to be an established risk factor for cardiovascular disease, current findings imply that this may be a modifiable risk factor, independent of other potential confounders, even among high-risk populations. It implies a more aggressive treatment to raise HDL-C will benefit patients by reducing the risk of $\mathrm{CV} / \mathrm{CB}$ events. Despite the large sample size and accounting of several potential confounders, further research among a more diverse population (both in demographics and concomitant medications) may be warranted to confirm these findings, further add to the breadth of evidence available on the impact of changes in HDL-C on $\mathrm{CV}$ and/or $\mathrm{CB}$ events, and further inform clinical practice.

\section{ACKNOWLEDGEMENTS}

The authors have no acknowledgements to disclose. Funding for this manuscript was provided by Merck and Co., Inc. Drs. Ambegaonkar, Bash and Sazonov are employees of Merck and Co., Inc.

\section{REFERENCES}

[1] The World Health Organization (2011) The World Health Organization: Cardiovascular diseases. http://www.who.int/mediacentre/factsheets/fs317/en/inde x.html\#

[2] British Heart Foundation (2010) Coronary heart disease statistics. http://www.bhf.org.uk/idoc.ashx?docid=9ef69170-3edf-4 fbb-a202-a93955c1283d\&version=-1

[3] The World Health Organization (2011) The World Health Organization: NCD country profiles: United States of America. http://www.who.int/nmh/countries/usa_en.pdf 
[4] Lloyd-Jones, D., Adams, R., Carnethon, M., et al. (2009) Heart disease and stroke statistics_-2009 update: A report from the American Heart Association Statistics Committee and Stroke Statistics Subcommittee. Circulation, 119, 480-486. doi:10.1161/CIRCULATIONAHA.108.191259

[5] U.S. Centers for Disease Control and Prevention (2011) National vital statistics reports. http://www.cdc.gov/nchs/data/nvsr/nvsr59/nvsr59_04.pdf

[6] Erhardt, L.R. and Gotto, A. Jr. (2006) The evolution of European guidelines: Changing the management of cholesterol levels. Atherosclerosis, 185, 12-20. doi:10.1016/j.atherosclerosis.2005.10.001

[7] Grundy, S.M., Cleeman, J.I., Merz, C.N., et al. (2004) Implications of recent clinical trials for the national cholesterol education program adult treatment panel III guidelines. Journal of the American College of Cardiology, 44, 720-732. doi:10.1016/j.jacc.2004.07.001

[8] Graham, I., Atar, D., Borch-Johnsen, K. et al. (2007) European guidelines on cardiovascular disease prevention in clinical practice: Executive summary. European Heart Journal, 28, 2375-2414. doi:10.1093/eurheartj/ehm316

[9] Sarwar, N., Danesh, J., Eiriksdottir, G., et al. (2007) Triglycerides and the risk of coronary heart disease: 10,158 incident cases among 262,525 participants in 29 western prospective studies. Circulation, 115, 450-458. doi:10.1161/CIRCULATIONAHA.106.637793

[10] Morrison, A. and Hokanson, J.E. (2009) The independent relationship between triglycerides and coronary heart disease. Vascular Health Risk Management, 5, 89-95.

[11] Gordon, T., Castelli, W.P., Hjortland, M.C., et al. (1977) High density lipoprotein as a protective factor against coronary heart disease: The Framingham study. American Journal of Medicine, 62, 707-714. doi:10.1016/0002-9343(77)90874-9

[12] Sharrett, A.R., Ballantyne, C.M., Coady, S.A., et al. (2001) Coronary heart disease prediction from lipoprotein cholesterol levels, triglycerides, lipoprotein(a), apolipoproteins A-I and B, and HDL-C density subfractions: The Atherosclerosis Risk in Communities (ARIC) study. Circulation, 104, 1108-1113. doi:10.1161/hc3501.095214

[13] Miller, M., Cannon, C.P., Murphy, S.A., et al. (2008) Impact of triglyceride levels beyond low-density lipoprotein cholesterol after acute coronary syndrome in the PROVE IT-TIMI 22 trial. Journal of American College of Cardiology, 51, 724-730. doi:10.1016/j.jacc.2007.10.038

[14] Faergeman, O., Holme, I., Fayyad, R., et al. (2009) Plasma triglycerides and cardiovascular events in the treating to new targets and incremental decrease in end-points through aggressive lipid lowering trials of statins in patients with coronary artery disease. American Journal of Cardiology, 104, 459-463. doi:10.1016/j.amjcard.2009.04.008

[15] Barter, P., Gotto, A.M., LaRosa, J.C., et al. (2007) HDLC cholesterol, very low levels of LDL cholesterol, and cardiovascular events. New England Journal of Medicine, 357, 1301-1310. doi:10.1056/NEJMoa064278

[16] Shanthi, M., Puska, P. and Norrving, B. (2011) Global Atlas on cardiovascular disease prevention and control. http://whqlibdoc.who.int/publications/2011/97892415643 73 _eng.pdf
[17] Gordon, T., Kannel, W.B., Castelli, W.P., et al. (1981) Lipoproteins, cardiovascular disease, and death: The Framingham study. Archives of Internal Medicine, 141, 11281131. doi:10.1001/archinte.1981.00340090024008

[18] Kini, A.S., Muntner, P., Moreno, P.R., et al. (2009) Relation of high-density lipoprotein cholesterol to mortality after percutaneous coronary interventions in patients with low-density lipoprotein $<70 \mathrm{mg} / \mathrm{dl}$. American Journal of Cardiolology, 103, 350-354. doi:10.1016/j.amjcard.2008.09.087

[19] Rywik, S.L., Manolio, T.A., Pajak, A., et al. (1999) Association of lipids and lipoprotein level with total mortality and mortality caused by cardiovascular and cancer diseases (Poland and United States collaborative study on cardiovascular epidemiology). American Journal of Cardiolology, 84, 540-548. doi:10.1016/S0002-9149(99)00374-4

[20] Ghazzal, Z.B., Dhawan, S.S., Sheikh, A., et al. (2009) Usefulness of serum high-density lipoprotein cholesterol level as an independent predictor of one-year mortality after percutaneous coronary interventions. American Journal of Cardiolology, 103, 902-906.

doi:10.1016/j.amjcard.2008.11.053

[21] Goldenberg, I., Benderly, M., Sidi, R., et al. (2009) Relation of clinical benefit of raising high-density lipoprotein cholesterol to serum levels of low-density lipoprotein cholesterol in patients with coronary heart disease (from the Bezafibrate infarction prevention trial). American Journal of Cardiolology, 103, 41-45. doi:10.1016/j.amjcard.2008.08.033

[22] Bittner, V., Johnson, B.D., Zineh, I., et al. (2009) The triglyceride/high-density lipoprotein cholesterol ratio predicts all-cause mortality in women with suspected myocardial ischemia: A report from the Women's Ischemia Syndrome Evaluation (WISE). Americn Heart Journal, 157, 548-555. doi:10.1016/j.ahj.2008.11.014

[23] Cui, Y., Watson, D.J., Girman, C.J., et al. (2009) Effects of increasing high-density lipoprotein cholesterol and decreasing low-density lipoprotein cholesterol on the incidence of first acute coronary events (from the Air Force/ Texas Coronary Atherosclerosis Prevention Study). American Journal of Cardiolology, 104, 829-834. doi:10.1016/j.amjcard.2009.05.020

[24] Arsenault B., Barter P., DeMicco D., et al. (2011) Prediction of cardiovascular events in statin-treated stable coronary patients by lipid and nonlipid biomarkers. Journal of American College of Cardiolology, 57, 63-69. doi:10.1016/i.jacc.2010.06.052

[25] Sazonov, V., Beetsch, J., Phatak, H., et al. (2010) Association between dyslipidemia and vascular events in patients treated with statins: Report from the UK General Practice Research Database. Atherosclerosis, 208, 210216. doi:10.1016/j.atherosclerosis.2009.07.021

[26] Walley, T. and Mantgani, A. (1997) The UK general practice research database. Lancet, 350, 1097-1099. doi:10.1016/S0140-6736(97)04248-7

[27] Jick, S.S., Kaye, J.A., Vasilakis-Scaramozza, C., et al. (2003) Validity of the general practice research database. Pharmacotherapy, 23, 686-689. doi:10.1592/phco.23.5.686.32205 
[28] Waters, D. (1999) Cholesterol lowering: Should it continue to be the last thing we do? Circulation, 99, 32153217.

[29] Grover, S., Kaouache, M., Joseph, L., et al. (2009) Evaluating the incremental benefits of raising high-density lipoprotein cholesterol levels during lipid therapy after adjustment for the reductions in other blood lipid levels.
Archives of Internal Medicine, 169, 1775-1780. doi:10.1001/archinternmed.2009.328

[30] Tziomalos, K., Athyros, V.G., Karagiannis, A., et al. (2009) Dyslipidemia as a risk factor for ischemic stroke. Current Topics in Medicinal Chemistry, 9, 1291-1297. doi: $10.2174 / 156802609789869628$ 